\title{
L'incidence axiale dans la luxation de la patella
}

\section{Skyline View in Patellar Dislocation}

\section{S. Chabrier · P. Kauffmann $\cdot$ P. Le Borgne}

Reçu le 6 janvier 2016; accepté le 5 février 2016

(C) SFMU et Lavoisier SAS 2016

Une femme de 18 ans s'est présentée aux urgences pour une douleur du genou droit survenue lors d'un match de badminton. Initialement, elle rapportait un craquement et une sensation de dérobement du genou lors d'un appui en pivot, cela associé à une impotence fonctionnelle majeure. L'examen clinique aux urgences retrouvait un épanchement articulaire $\mathrm{du}$ genou droit, avec comblement du récessus sousquadricipital, et une zone douloureuse élective le long du bord médial de la patella. La radiographie de face et de profil ne retrouvait aucune lésion visible. L'incidence fémoropatellaire (FP) à $30^{\circ}$ retrouvait un défect osseux de la face articulaire médiale de la patella ainsi qu'un fragment osseux au fond du récessus latéropatellaire correspondant à une fracture ostéochondrale (Fig. 1). La localisation de cette fracture oriente vers une luxation latérale de la patella réduite spontanément. Celle-ci survient préférentiellement chez les adultes jeunes, en général avec un terrain favorisant comme une dysplasie FP ou une hyperlaxité ligamentaire. Le mécanisme lésionnel correspond le plus souvent à un traumatisme indirect associant valgus, flexion et rotation latérale. Les antécédents familiaux identiques, la localisation de la douleur au bord médial de la patella (zone de déchirure préférentielle du ligament FP médial) et une hémarthrose doivent faire évoquer ce diagnostic de luxation de la patella réduite spontanément et imposent la recherche systématique d'une fracture ostéochondrale. Elle fait suite à l'impaction de la patella sur le condyle et se situe le plus souvent à la face médiale de la patella, mais également parfois sur le condyle fémoral latéral. Elle est omise dans 30 à $40 \%$ des radiographies initiales, l'incidence FP à $30^{\circ}$ doit faire systématiquement partie du bilan radiographique initial dans ce contexte évocateur, afin

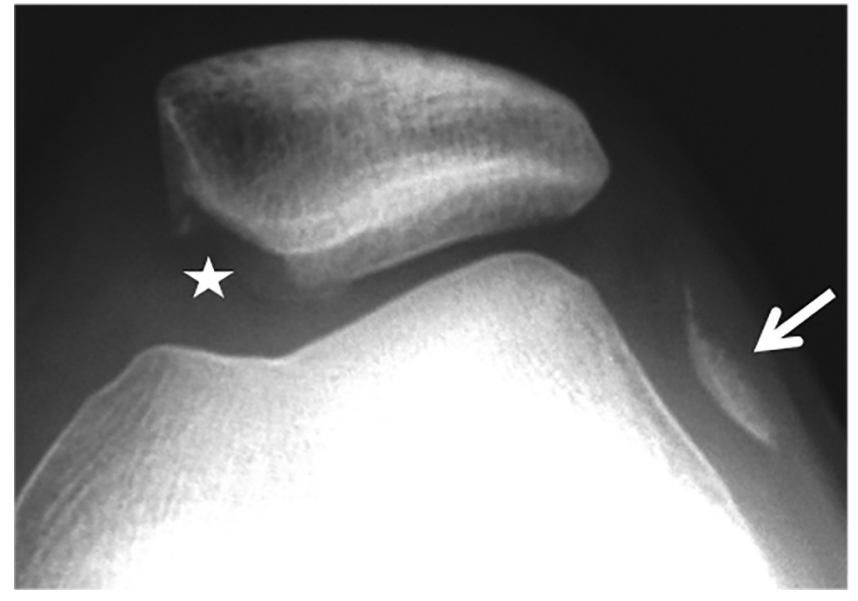

Fig. 1 Radiographie du genou droit, incidence fémoropatellaire à $30^{\circ}$. Étoile : défect osseux de la face médiale de la patella. Flèche : fragment ostéochondral

d'améliorer le dépistage de cette lésion [1]. Négligées, ces fractures peuvent donner des douleurs résiduelles, des blocages articulaires et avoir une évolution arthrosique précoce. La patiente a ici bénéficié d'une arthroscopie avec fixation du fragment par une minivis et ne présentait aucune séquelle à trois mois.

\section{Référence}

1. Metzler AV, Lattermann C, Johnson DL (2015) Cartilage lesions of the patella: management after acute patellar dislocation. Orthopedics 38:310-4.

S. Chabrier · P. Kauffmann · P. Le Borgne $(\bowtie)$

Service d'accueil des urgences,

hôpitaux universitaires de Strasbourg, hôpital de Hautepierre,

1, avenue Molière, F-67098 Strasbourg, France

e-mail : pierrick_med@yahoo.fr

P. Le Borgne

Faculté de médecine, université de Strasbourg,

4, rue Kirschleger, F-67000 Strasbourg, France 